\title{
The Pierre Auger Observatory status and the AugerPrime upgrade program
}

\author{
Daniele Martello ${ }^{1, a}$ on behalf of the Pierre Auger Collaboration ${ }^{2, b}$ \\ 1 Dipartimento di Matematica e Fisica "E. De Giorgi” and INFN Lecce, via per Arnesano, Lecce, Italy \\ 2 Observatorio Pierre Auger, Av. San Martin Norte, 304, 5613 Malargue, Argentina
}

\begin{abstract}
The nature and the origin of ultra-high energy cosmic rays (UHECRs), above $10^{17} \mathrm{eV}$, are still unknown. The Pierre Auger Observatory with its huge exposure provides us with a large set of high quality data. The analysis of these data has led to major breakthroughs in the last decade, but a coherent interpretation is still missing. To answer the open questions the Observatory has started a major upgrade, with an emphasis on improved mass composition determination using the surface detectors. The latest results and the planned detector upgrade will be presented. The expected performance and the improved physics sensitivity of the Observatory will be discussed.
\end{abstract}

\section{Introduction}

The nature and the origin of ultra-high energy cosmic rays above $10^{17}$ are still unknown, even if in the last decade measurements have shed light on these puzzling questions. Understanding the sources, nature and propagation properties of UHECRs is one of the key questions in astroparticle physics. From the experimental point of view, their study can be performed indirectly, by using the extensive air showers (EASs) they produce by interacting with the nuclei of the molecules that compose the atmosphere of the Earth.

Anyway, the all particle spectrum by itself cannot provide sufficient discrimination between the different astrophysical hypotheses, and the determination of the primary composition is mandatory to reach any reliable conclusion.

\section{The Pierre Auger Observatory}

The Pierre Auger Observatory [1] with its $3000 \mathrm{~km}^{2}$ of instrumented surface, brings unique capabilities to the UHECR study. The Observatory is located in the province of Mendoza (Argentina) near the city of Malargue in a vast high plain. It is the world's largest cosmic ray observatory. The Observatory consists of an array (SD) of 1600 waterCherenkov particle detector stations (WCD) overlooked by 24 air fluorescence telescopes (FD). In addition, three high elevation fluorescence telescopes (HEAT) overlook a surface of $23.5 \mathrm{~km}^{2}$ where 61 additional WCDs (Infill) are installed (see Fig. 1).

Each WCD of the surface detector is a waterCherenkov detector which samples the particle content of the EAS falling on the array. The WCD consists of a 12,000 liter polyethylene water tank containing a

\footnotetext{
a e-mail: daniele.martello@le.infn.it

b e-mail: auger_spokespersons@fnal.gov

http://www . auger.org/archive/authors_2016_09.html
}

sealed laminated polyethylene liner with a reflective inner surface. Cherenkov light from the passage of charged particles is collected by three $230 \mathrm{~mm}$ photomultiplier tubes (PMTs) that look through windows of clear polyethylene into highly purified water. The WCD is self contained. A solar power system provides power for the PMTs and electronics package. The electronics package, consisting of a processor, GPS receiver, radio transceiver and power controller, is mounted on the container. The WCD stations are placed on a triangular grid with $1500 \mathrm{~m}$ spacing (SD-1500).

The 24 telescopes of the FD overlook the SD array from four sites: Los Leones, Los Morados, Loma Amarilla and Coihueco [1] (see Fig. 1). Six independent telescopes are located at each FD site in a clean climate-controlled building. A single telescope has a field of view of $30^{\circ} \times$ $30^{\circ}$ in azimuth and elevation, with a minimum elevation of $1.5^{\circ}$ above the horizon. The telescopes face towards the interior of the array so that the combination of the six telescopes provides $180^{\circ}$ coverage in azimuth.

Three additional fluorescence telescopes (HEAT) with an elevated field of view were built at the FD site at Coihueco. These telescopes are very similar to the original fluorescence telescopes but are tilted by $29^{\circ}$ upward. These three telescopes work independently of other FD sites and form the "fifth site" of the Observatory. The HEAT telescopes were designed to cover the elevation range from $30^{\circ}$ to $58^{\circ}$, which lies above the field of view of the other FD telescopes. The HEAT telescopes allow a determination of the cosmic ray spectrum and $X_{\max }$ distributions in the energy range from below the second knee up to the ankle.

The Infill array consists of a denser WCD array with $750 \mathrm{~m}$ spacing (SD-750) nested within the $1500 \mathrm{~m}$ array covering a surface of $23.5 \mathrm{~km}^{2}$. The area is centered $6 \mathrm{~km}$ away from the Coihueco fluorescence site. The Infill array is fully efficient from $3 \times 10^{17} \mathrm{eV}$ onwards for air showers with zenith angle $\leq 55^{\circ}$. 


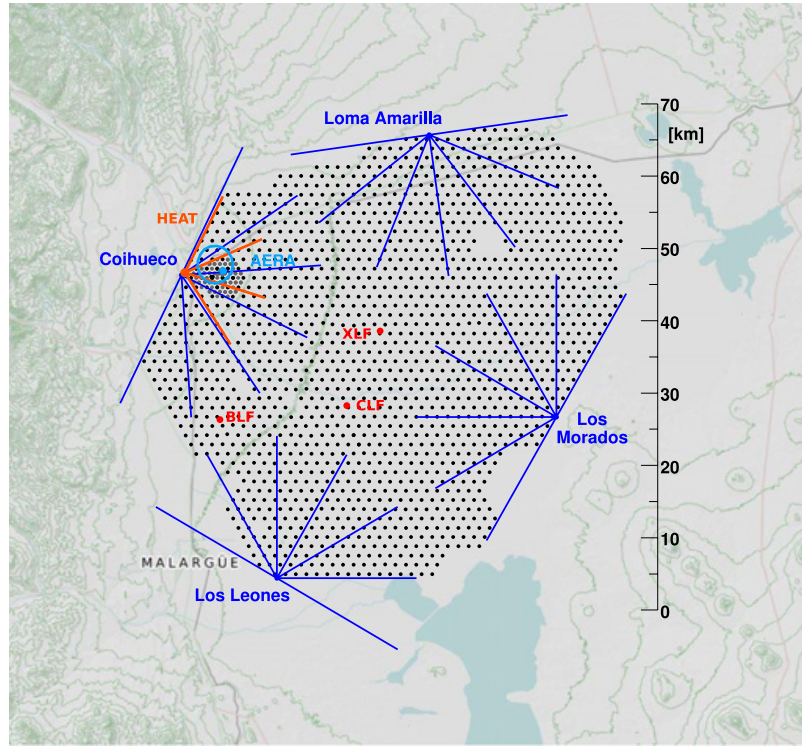

Figure 1. The Auger Observatory layout. Each dot corresponds to one of the 1660 surface detector stations. The four fluorescence detector sites are shown, each with the field of view of its six telescopes. The Coihueco site hosts three extra high elevation (HEAT) telescopes. The $750 \mathrm{~m}$ array is located a few kilometers from Coihueco.

On-line and long-term performance of the detectors and data quality are monitored continuously, and a set of high-quality devices installed in the Observatory monitors the atmospheric conditions during operation.

High-quality data have been collected continuously for about ten years, with a SD annual exposure of $5500 \mathrm{~km}^{2}$ sr yr. The longitudinal profile reconstructed by the FD is providing a nearly calorimetric measurement of the primary energy, with total systematic uncertainty of $14 \%$ [2]. From the shower lateral distribution reconstructed using the WCD signals, a SD energy estimator is inferred. A high-quality subset of hybrid events recorded by both the $\mathrm{SD}$ and the FD is used to calibrate the SD energy estimator with the FD energy measurement, hence providing an almost model-independent energy calibration.

\section{The spectrum}

The Pierre Auger Observatory has collected data of excellent quality for more than 10 years, which has already led to a measurement of the flux of UHECRs above $3 \times$ $10^{17} \mathrm{eV}$ with unprecedented statistics.

The events used for the determination of the energy spectrum consist of 4 different sets of data: the SD-1500 vertical events with zenith angle up to $60^{\circ}$, the SD-1500 inclined events with zenith angle between $60^{\circ}$ and $80^{\circ}$, the SD-750 vertical events and the hybrid events. The hybrid set of data contains events detected simultaneously by the fluorescence telescopes and by at least one WCD.

The first step in the procedure used for the determination of the spectrum is the evaluation of the energy of the events. The FD allows the measurement of the electromagnetic energy released by the shower in the atmosphere as a function of the atmospheric depth. The total primary energy is then derived by integrating this longitudinal profile over the depth range and adding an

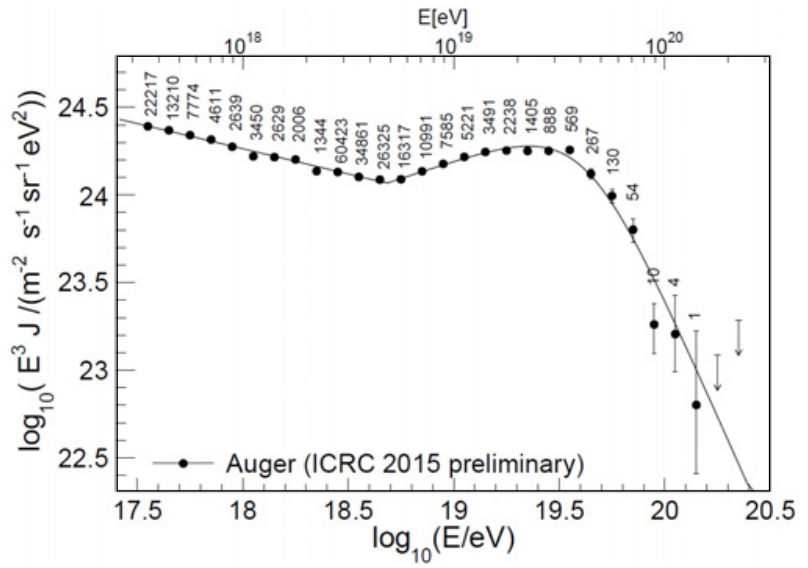

Figure 2. The combined energy spectrum measured by the Auger Observatory, fitted with a flux model. The data points include only the statistical uncertainties. For each data point is reported the number of events.

estimate of the so-called "invisible energy" carried into the ground by high-energy muons and neutrinos.

The SD samples the shower particles that reach the ground. The intensities of the signals registered in the WCD are used to quantify the shower size and the impact point of the shower axis on the ground.

The absolute calibration of the SD sets of data is inferred from a high-quality subset of hybrid events (full details in [3-5]).

The final step in the procedure used for the determination of the energy spectrum is a precise evaluation of the exposure. Above the energy for full detector efficiency, the calculation of the SD exposure is based on the determination of the geometrical aperture of the array for the corresponding zenith-angle interval and of the observation time. The choice of a fiducial trigger based on active hexagons allows one to exploit the regularity of the array, and to compute the aperture simply as the sum of the areas of all active hexagons. The calculation of the exposure for the hybrid set of data is more complex. It relies on a detailed time-dependent Monte Carlo simulation which exactly reproduces the data taking conditions and includes the response of the hybrid detector [6].

The energy spectrum reported in Fig. 2 has been obtained by combining the four independent sets of data. They are combined using a method that takes into account the systematic uncertainties of the individual measurements (see details in [7]).

The characteristic features of the combined energy spectrum, shown in Fig. 2, have been quantified by fitting a model that describes a spectrum by a power-law below the ankle $J(E)=J_{0}\left(E / E_{\text {ankle }}\right)^{\gamma_{1}}$ and power-law with a smooth suppression at the highest energies:

$$
\begin{aligned}
J(E)= & J_{0}\left(\frac{E}{E_{\text {ankle }}}\right)^{-\gamma_{2}}\left[1+\left(\frac{E_{\text {ankle }}}{E_{s}}\right)^{\Delta \gamma}\right] \\
& \times\left[1+\left(\frac{E}{E_{s}}\right)^{\Delta \gamma}\right]^{-1}
\end{aligned}
$$

Here, $\gamma_{1}$ and $\gamma_{2}$ are the spectral indexes below and above the ankle energy $E_{\text {ankle }}$ respectively, $E_{s}$ is the energy at which the differential flux falls to one-half of the value of 

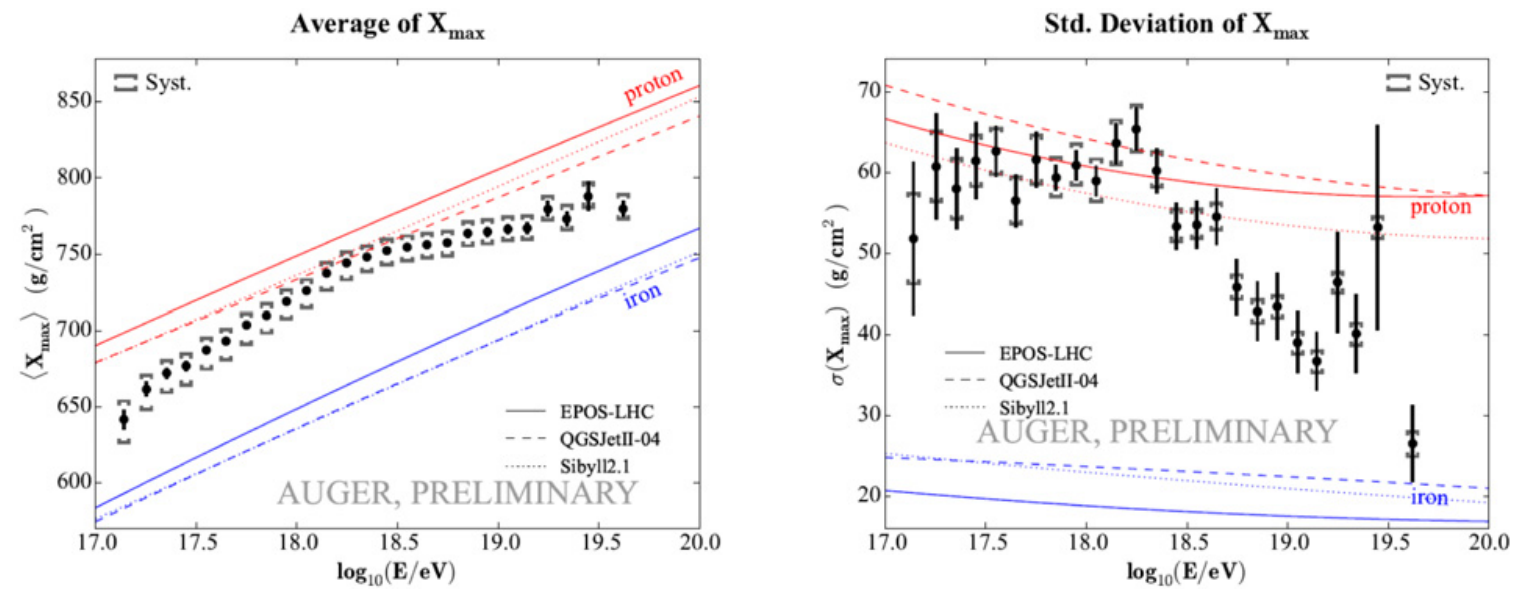

Figure 3. Average and RMS of the $X_{\max }$ compared to the model predictions for an all-proton and an all-iron composition.

Table 1. Best-fit parameters for the combined energy spectrum, statistical and systematic uncertainties being reported.

\begin{tabular}{llllll}
\hline$J_{0}\left[\mathrm{eV}^{-1} \mathrm{~km}^{-2} \mathrm{sr}^{-1} \mathrm{yr}^{-1}\right]$ & $E_{\text {ankle }}[\mathrm{EeV}]$ & $E_{s}[\mathrm{EeV}]$ & $\gamma_{1}$ & $\gamma_{2}$ & $\Delta \gamma$ \\
\hline$(3.30 \pm 0.15 \pm 0.20) \times 10^{-19}$ & $4.82 \pm 0.07 \pm 0.8$ & $42.1 \pm 1.7 \pm 7.6$ & $3.29 \pm 0.02 \pm 0.05$ & $2.69 \pm 0.02 \pm 0.1$ & $3.14 \pm 0.2 \pm 0.4$ \\
\hline
\end{tabular}

the power-law extrapolation from the intermediate region, $\Delta \gamma$ gives the increment of the spectral index beyond the suppression region, and $J_{0}$ is the normalization of the flux, taken as the value of the flux at $E=E_{\text {ankle }}$.

The result of the best fit is shown in Fig. 2 and the corresponding parameters are presented in Table 1, quoting both statistical and systematic uncertainties.

The energy spectrum can also be exploited to study the distribution of cosmic-ray sources by searching for a flux variation with declination $(\delta)$ of the incoming directions. This study is of particular interest to the discussion of the difference seen in the suppression region between the spectra measured by Auger and by the Telescope Array experiment [8], which, despite being still compatible within the quoted systematic uncertainties of both experiments, is not understood so far.

\section{Mass composition}

Different observables can be used to obtain information on the primary composition, the most direct of which is the depth of maximum development of the longitudinal shower profile $\left(X_{\max }\right)$, measured by the FD. $X_{\max }$ is related to the depth of the first interaction of the primary and to the subsequent development of the shower. For this reason, the interpretation in terms of composition is complicated by the large uncertainties in the hadronic interaction models used in the simulations (see [9] these proceedings). The average of the $X_{\max }$ for different energies of the primary and its RMS can be directly compared to the predictions of air shower simulations using recent post-LHC hadronic interaction models, as shown in Fig. 3.

Our measurements are clearly at variance with model predictions for pure composition; assuming no change in hadronic interactions at these energies, they point to a composition getting heavier above the ankle.

\section{Search for Anisotropy}

Complementary to the spectrum and mass measurements are the searches for anisotropy. Due to the energy losses during propagation through the Cosmic Microwave Background (CMB) the position of the astrophysical sources of UHECRs should be relatively nearby, which in case of light nuclei with $E>40 \mathrm{EeV}$ would result in anisotropies in arrival directions, reflecting the distribution of the nearby extragalactic matter. For protons of this energy, deflections in the encountered magnetic fields are quite small, while for nuclei of atomic number $\mathrm{Z}$ they are $\mathrm{Z}$ times larger.

In the more recent analysis [10] 602 events have been selected with energy above $40 \mathrm{EeV}$ recorded in the 10 years of operation of the Pierre Auger Observatory in the declination range of $-90^{\circ}$ to $+45^{\circ}$. Various kinds of tests were performed to search for anisotropy above a certain threshold in various angular windows as well as autocorrelations of arrival directions. Correlations were also sought with catalogs of plausible candidates of UHECR sources. The two largest departures from isotropy are found above $58 \mathrm{EeV}$ and correspond to the direction within $15^{\circ}$ of Centaurus A, the closest radio-loud AGN, and to the arrival direction within $18^{\circ}$ of Swift-BAT AGNs closer than $130 \mathrm{Mpc}$ and brighter than $10^{44} \mathrm{erg} \mathrm{s}^{-1}$. None of the excesses is statistically significant (see Fig. 4). Therefore, either the primaries are light nuclei and the sources are numerous or the isotropy is caused by large deflections of nuclei with larger Z. A full sky study of arrival directions has been performed in collaboration with Telescope Array and IceCube [11]. The study included 231 events with energy above $52 \mathrm{EeV}$ from the Pierre Auger Observatory, 87 events with energy above $57 \mathrm{EeV}$ from the Telescope Array and the very high energy neutrinos from IceCube. No correlations at the discovery level were found.

Large scale anisotropy can reveal the effects of the global distribution of sources and the collective motion of cosmic rays. Using events recorded by the SD from 2004 January 1 to 2013 December 31 an analysis [12] was performed including also inclined events, reaching a coverage of about $85 \%$ of the sky. The set of data consists of about 70000 events with energies above $4 \mathrm{EeV}$, where full efficiency for inclined events is attained. Two Rayleigh 


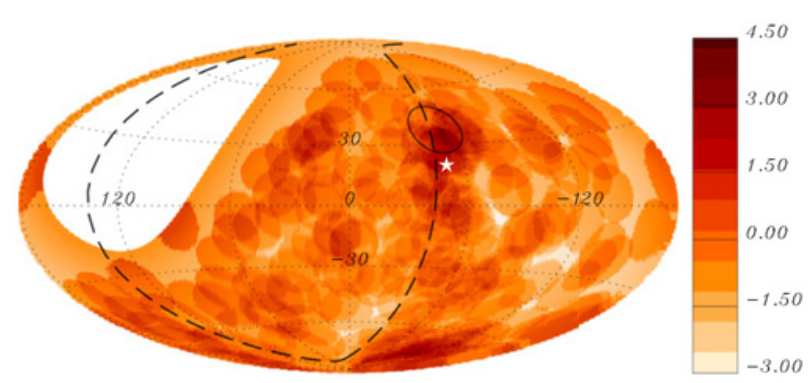

Figure 4. Map of the Li-Ma significances of overdensities in windows of $12^{\circ}$ for events with $E \geq 54 \mathrm{EeV}$. Dashed line: SuperGalactic Plane. White star: Centaurus A.
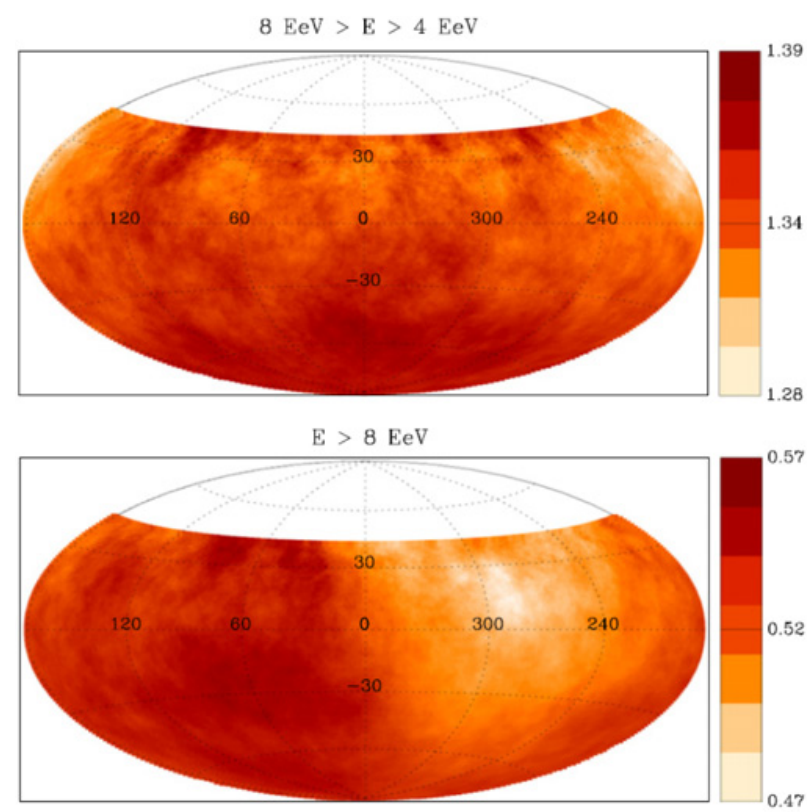

Figure 5. Sky map of flux for two bins of energy in $\mathrm{km}^{-2} \mathrm{yr}^{-1} \mathrm{sr}^{-1}$ units. The data are smoothed in angular windows of $45^{\circ}$. (Galactic coordinates).

analyses were performed, in right ascension and azimuth angles in two energy bins, 4-8 EeV and above $8 \mathrm{EeV}$. While the lower energy bin does not show any significant departure from isotropy of arrival directions, a dipole was found at energies above $8 \mathrm{EeV}$ of an amplitude $0.073 \pm$ 0.015 pointing to $(\alpha, \delta)=\left(-95^{\circ} \pm 13^{\circ},-39^{\circ} \pm 13^{\circ}\right)$. A sky map of the flux in equatorial coordinates is shown in Fig. 5. Observation of dipolar amplitudes in arrival directions of UHECRs is consistent with expectations for heavier nuclei suggested by the $X_{\max }$ distributions.

\section{Astrophysical interpretation}

The accurate measurement of the spectrum, the results from the study of the mass composition and the distribution of the arrival directions of the primaries, gives the possibility to infer some hypothesis on the origin and propagation of UHECRs [13].

Assuming that all UHECR sources emit hydrogen-1, helium-4, nitrogen-14 and iron-56 with a broken exponential rigidity cutoff, it is possible to infer some characteristics of the acceleration sites and put some constraint on the propagation process. Unfortunately, with the publicly available codes that simulate the propagation of the UHECRs from the sources to the Earth $[14,15]$, it is not possible to take into account the anisotropy study in the global fit procedure. For this reasons all the UHECR sources are assumed identical and uniformly distributed in the near universe. In this analysis both the propagation codes and different sets of parameters for the propagation processes are taken into account. The interaction of the primary with the atmosphere has been simulated with three different interaction codes [16-18]. The detector resolutions and acceptances are included. The data we attempt to fit consist of 15 measurements of the UHECR energy spectrum and 110 non-zero measurements of the $X_{\max }$ distribution. The free parameters of the fit are: the injection normalization factor $J_{0}$, the injection spectral index $\gamma$, the cutoff rigidity $R_{\text {cut }}$ and the fractions of the different primaries at injection (three free parameters). In total there are 125 non-zero data points and 6 free parameters.

The minimization of the $\chi^{2}$ distribution identifies a best fit solution for $\gamma=0.94_{-0.10}^{+0.09}$ and $R_{\text {cut }}=10^{18.67 \pm 0.03}$ with $\chi^{2} /$ d.o.f. $=178.5 / 119$. A second minimum in the $\chi^{2}$ distribution is found at $\gamma=2.03$ and $R_{\text {cut }}=10^{19.84}$ for $\chi^{2} /$ d.o.f. $=235 / 119$ [13]. The corresponding simulated spectra and the mean and variance of the simulated $X_{\max }$ distributions are shown in Fig. 6 for the two solutions. The best fit solution of the Auger data predicts a very hard injection spectrum $(\gamma<1)$ and a rigidity cutoff that implies that the UHECR flux above $10^{19.5} \mathrm{eV}$ is mostly limited by the maximum energy at the sources. The second minimum $(\gamma \sim 2)$ with its larger rigidity cutoff is more in line with the standard models for UHECR acceleration.

In this analysis, the spectrum measured with high statistics and with different combinations and configurations of detectors has been combined with the mass composition sensitive parameters detected only with the fluorescence detector in a limited range of energy and with a number of events that is about $15 \%$ of the statistics collected for the spectrum. Moreover, a similar analysis limited to regions of the sky where there is an evidence for anisotropies in the cosmic ray flux is desirable. This is not possible with the mass sensitive data collected only by the fluorescence detector due to the limited statistics.

\section{AugerPrime motivations}

In order to solve the uncertainty still present in the interpretation of the cosmic ray cutoff ([19]) an upgrade of the Auger Observatory (named AugerPrime [20,21]) has been planned. The main aim of AugerPrime is to provide additional measurements of observables sensitive to the mass composition of the most energetic cosmic rays, allowing the determination of the primary mass on a shower-by-shower basis. With AugerPrime it will be possible to study the mass composition not only with the fluorescence detector but also with the surface detector. The study of the origin of the flux suppression will provide fundamental constraints on the astrophysical sources, but also will allow us to determine more precise estimates of gamma-ray and neutrino fluxes at ultra-high energy. The high statistics of surface detector measurements will allow us to analyze the mass composition in different regions of the sky. Moreover, the AugerPrime upgrade will give us the possibility to measure the flux contribution of protons and elucidate the physics potential of existing and future cosmic ray, neutrino, and gamma-ray detectors. 

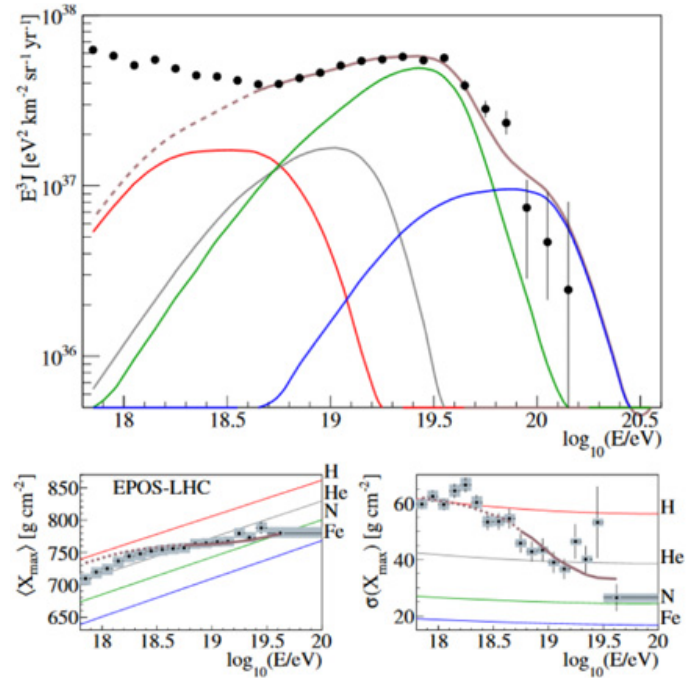
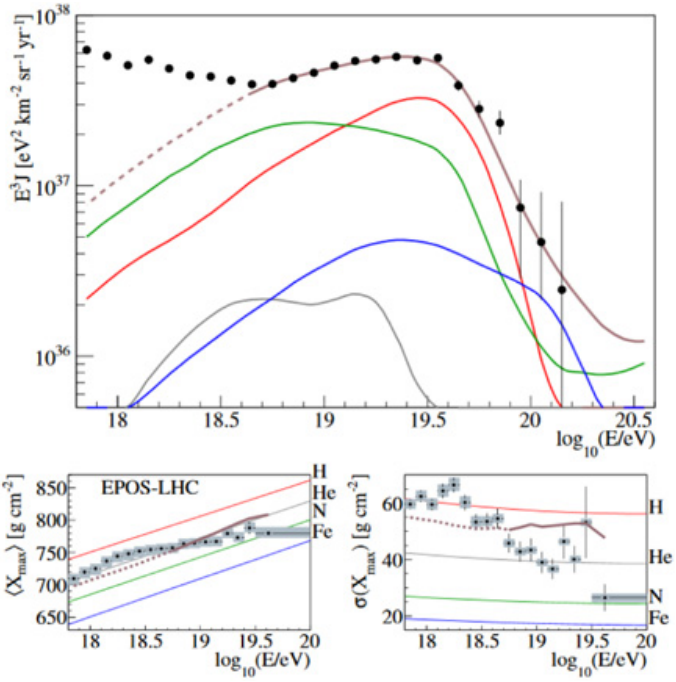

Figure 6. Top: simulated energy spectrum of UHECRs (multiplied by $E^{3}$ ) at the top of the Earths atmosphere with the best-fit parameters (left) and the second minimum of the likelihood for $\gamma \sim 2$ (right), compared with Auger data points. Partial spectra are grouped according to the mass number as follows: $\mathrm{A}=1$ (red), $2<\mathrm{A}<4$ (grey), $5<\mathrm{A}<26$ (green), $27<\mathrm{A}$ (blue), total (brown). Bottom: average and standard deviation of the $X_{\max }$ distribution as predicted in the two scenarios (brown). Pure $1 \mathrm{H}$ (red), $4 \mathrm{He}$ (grey), $14 \mathrm{~N}$ (green) and $56 \mathrm{Fe}$ (blue) are shown as reference. Only the energy range where the brown lines are solid is included in the fit.

The determination of the primary mass composition of ultra-high energy cosmic rays is also deeply related to our understanding of extensive air showers and hadronic interactions (see [9] these proceedings). In the Auger data, there is a disagreement between the observed and expected muon numbers, therefore it is important to study the hadronic interaction in extensive air showers.

\section{The AugerPrime upgrade}

The AugerPrime upgrade consists of four main improvements explained in the following subsections.

\subsection{The scintillator surface detector (SSD)}

The scintillator surface detector consists of a plane of scintillation detector mounted above and triggered by the larger WCD detector below it. The combination of the two detectors allows us to obtain a good measurement of the density of muons in the air showers.

An SSD unit is a box of area $3.8 \mathrm{~m} \times 1.3 \mathrm{~m}$, containing two scintillator sub-modules, each composed of extruded polystyrene scintillator bars of about $1.6 \mathrm{~m}$ length, $5 \mathrm{~cm}$ width and $1 \mathrm{~cm}$ thickness [21]. The $4 \mathrm{~m}^{2}$ scintillator planes are protected by light-tight, weatherproof enclosures, and mounted on top of the existing WCD with a strong support frame (see Fig. 7). The scintillator light will be read out with wavelength-shifting fibers inserted into straight extruded holes in the scintillator planes. The fibers are bundled and attached to a single light detector.

\subsection{The underground muon detector (AMIGA)}

The underground muon detector will provide a direct measurement of the muon content of a sample of showers observed by the upgraded Auger surface detector [22]. AMIGA will be used to verify and fine-tune the methods used to extract shower muon content using the SSD and WCD stations. AMIGA consists of 61 muon detectors deployed on a $750 \mathrm{~m}$ grid in the Infill area of the SD,

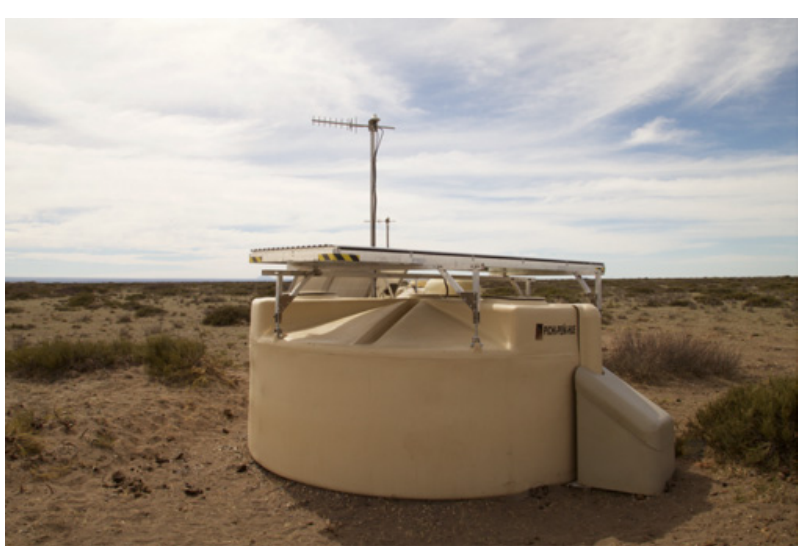

Figure 7. One SSD module already installed in the Pierre Auger Observatory.

instrumenting a total area of $23.5 \mathrm{~km}^{2}$. Each detector consists of a plane of plastic scintillator of about $30 \mathrm{~m}^{2}$ that will be buried about 2 meters underground.

\subsection{Change in fluorescence detector operation}

The fluorescence detector [1] provides information about extensive air showers such as a model-independent energy reconstruction and longitudinal development profiles of the extensive air showers. The main limitation of the FD is its duty cycle, currently at the level of $15 \%$. A significant increase of the duty cycle is possible by the extension of the FD operation to times at which a large fraction of the moon in the sky is illuminated. However, during such operations the PMT gain must be reduced by lowering the supplied high voltage (HV) to avoid high anode current and, therefore, an irreversible deterioration of the PMT sensitivity. The HV power used for the FD allows switching between two high voltage levels and the PMTs can be operated at the nominal gain (standard operation mode) and a lower gain (new operation mode with a large fraction of the moon in the sky). 

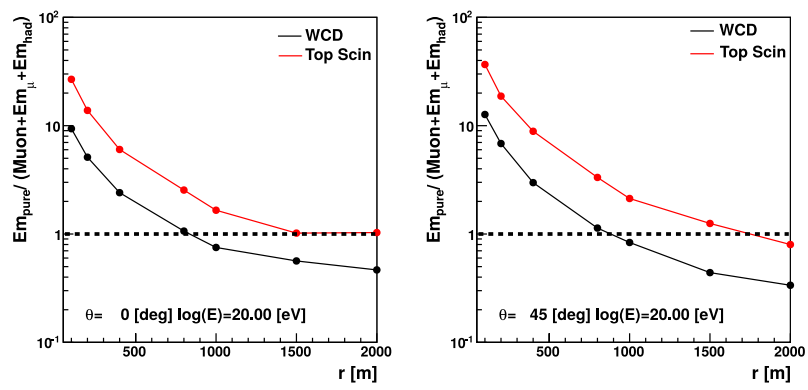

Figure 8. Ratios of different contributions to the integrated signal detected for air showers of $10^{20} \mathrm{eV}$ at two zenith angles. Shown is the ratio between the electromagnetic component and the muonic component. The curve labeled "WCD" corresponds to the waterCherenkov detectors of the Auger array, while the red one "Top Scin" corresponds to the scintillator detector.

\section{AugerPrime performance}

A thin scintillation detector, which is mounted above the larger water-Cherenkov detector, provides a robust and well-understood scheme for particle detection that is sufficiently complementary to the water-Cherenkov technique and permits a good measurement of the density of muons. This can be understood by comparing the signal contributions for different shower components as shown in Fig. 8. Over a wide range in lateral distance, the ratio between the integrated signal of electromagnetic particles (photons and electrons) and that of muons is more than a factor two higher in a scintillation detector without any shielding than in a water-Cherenkov detector.

The matrix formalism developed in [23] for a layered surface detector can be used for the reconstruction of the number of muons detected in a single upgraded detector station. The signal responses to particles of the muonic and electromagnetic shower components in the two detectors allow us to derive the muonic signal according to the expression:

$$
S_{\mu, W C D}=a S_{W C D}+b S_{S S D}
$$

where $S_{\mu, W C D}$ is the muonic signal in the WCD that can be converted in number of vertical equivalent muons (VEMs). $S_{W C D}$ and $S_{S S D}$ are respectively the total signal in the WCD and the total signal in the SSD. $a$ and $b$ are parameters inferred from detector simulation and are weakly dependent on the lateral distance from the shower core and on the mass of the primary.

To check the impact of this small dependence, the resolution and the mass-dependent bias in the reconstruction of the muon density for individual detector stations are given in Fig. 9. If stations with a lateral distance up to $1000 \mathrm{~m}$ are considered, the resolution is about $20-30 \%$ at the single detector level for showers in the flux suppression region. It is expected that the resolution can be improved for large lateral distances by using the constraint that the detector signal is dominated there by muons.

Another method that can be used to separate the muonic from the electromagnetic signals in the upgraded $\mathrm{SD}$ is based on the universality principle [24].

A universality-based analysis, or a sophisticated multivariate analysis, allows us to correlate the detector signals at different lateral distances and also takes

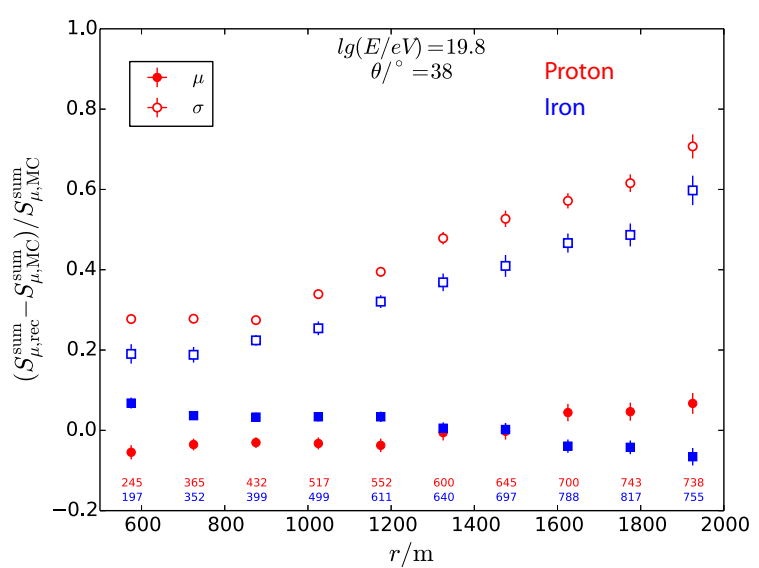

Figure 9. Reconstruction bias (solid symbols) and resolution (open symbols) of the muonic signal contribution for individual detector stations. The results for proton and iron showers are shown in red and blue, respectively. The number of detector stations analyzed for the different lateral distance intervals is also given.

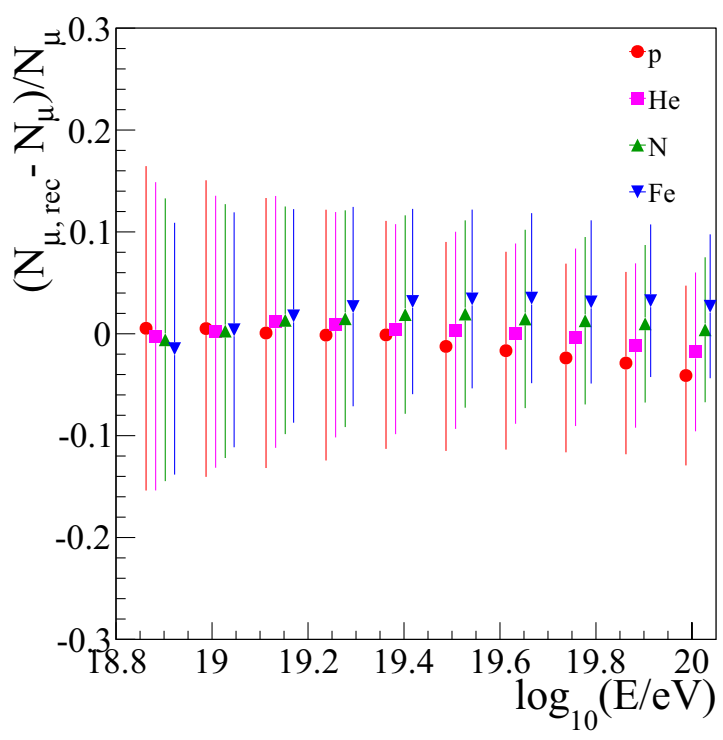

Figure 10. The reconstructed number of muons compared with the true number of muons as a function of energy for different cosmic ray primaries. Error bars represent the RMS of the distributions.

advantage of the arrival time (shower front curvature) and temporal structure of the signal measured in the detectors. The universality method predicts for the entire range of primary masses the air shower characteristics on the ground using only three parameters: the shower energy, the depth of shower maximum and the number of muons. Therefore, analyzing the structure of the signals in a ground based detector it is possible to infer the three parameters. The redundancy coming from the resampling of almost the same particles in two independent detectors (WCD and SSD) permits to eliminate possible degeneracies thanks to the different response to the muonic and electromagnetic components of the two detectors (see Fig. 8). The bias of the $X_{\max }$ reconstruction is less than $15 \mathrm{~g} / \mathrm{cm}^{2}$ with a resolution improving from $40 \mathrm{~g} / \mathrm{cm}^{2}$ at $10^{19} \mathrm{eV}$ to $25 \mathrm{~g} / \mathrm{cm}^{2}$ at $10^{20} \mathrm{eV}$. The corresponding results for the muon number reconstruction are shown in Fig. 10. 

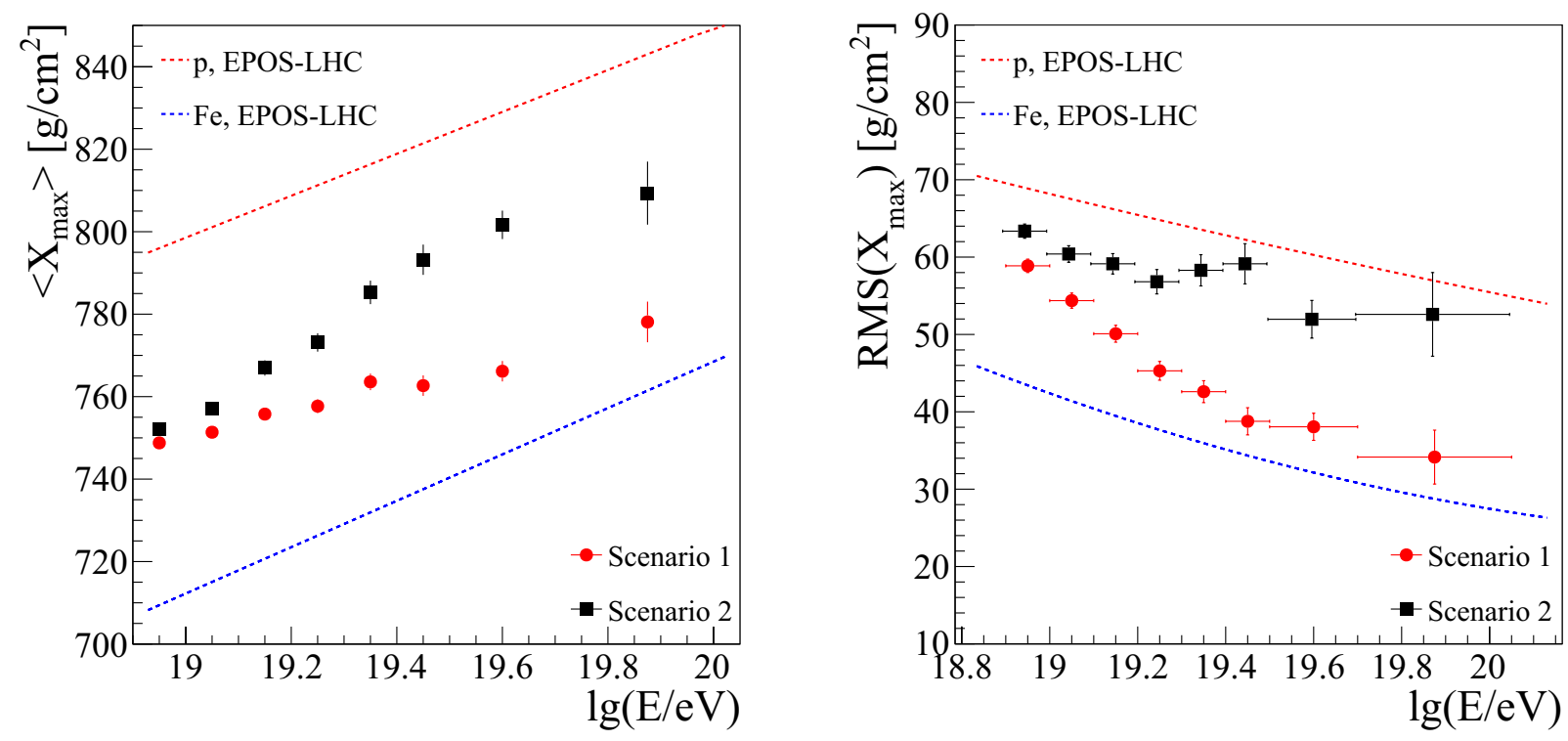

Figure 11. Predicted $X_{\max }$ and $\sigma\left(X_{\max }\right)$ for the two scenarios in Sect. 6 .

\section{AugerPrime expected science impact}

One of the key questions of the Pierre Auger upgrade is that of being able to discriminate between different compositions and, hence, physics scenarios in the energy range of the flux suppression. This is very difficult to demonstrate without knowing what composition to expect. For this reason two benchmark spectra have been chosen as representations of a maximum-rigidity scenario (scenario 1 corresponding to the best fit solution, Sect. 6) and of a photo-disintegration scenario (scenario 2 corresponding to the second minimum, Sect. 6).

Figure 11 shows the mean $X_{\max }$ and the corresponding $\sigma\left(X_{\max }\right)$ for these spectra, using the SD data of the upgraded array. The $\sigma\left(X_{\max }\right)$ contains the intrinsic airshower fluctuations and the detector resolution. The same quantities as expected for pure proton and pure iron compositions are illustrated. The difference in the evolution of these two observables for the two models in the energy range of the cutoff can be distinguished with high significance. While the mean $X_{\max }$ and $\sigma\left(X_{\max }\right)$ are very similar up to $10^{19.2} \mathrm{eV}$, which corresponds to the energy range covered by data of the fluorescence telescopes, the models predict significantly different extrapolations into the GZK suppression region and the two scenarios can be distinguished with high significance and statistics. In addition to these studies, the availability of muon information on an event-by-event basis allows for many ways of studying features of hadronic interactions. For example, the correlation between the number of muons and the depth of shower maximum can be used to study general features of muon production, including the search for exotic interaction scenarios at very high energy.

\section{Conclusions}

AugerPrime will collect high-quality data from 2018 until 2024. The statistic collected will be double compared with the existing Pierre Auger data sets, with the advantage that every event will have mass information and will allow us to better address some of the most pressing questions in UHECR physics. Obtaining additional compositionsensitive information will help to better reconstruct the properties of the primary particles at the highest energies. Moreover, it will improve the measurements in the important energy range just above the spectral ankle. Measurements with the new detectors will help to reduce systematic uncertainties related to the hadronic interaction models and to the reconstruction algorithms. This improved knowledge of air-shower physics will allow a reanalysis of existing data for improved energy evaluation and for improved mass composition studies.

\section{References}

[1] The Pierre Auger Collaboration, Nucl. Instrum. Meth. A 798, 172 (2015)

[2] V. Verzi for the Pierre Auger Collaboration, in Proc. of ICRC 2013 (Proceedings, Rio de Janeiro Brazil, 2013) 0928

[3] The Pierre Auger Collaboration, JCAP 1408, 08, 019 (2014)

[4] R. Pesce for the Pierre Auger Collaboration, in Proc. of ICRC 2011 (Proceedings, Beijing China, 2011) 0214

[5] D. Ravignani for the Pierre Auger Collaboration, in Proc. of ICRC 2013 (Proceedings, Rio de Janeiro Brazil, 2013) 0693

[6] The Pierre Auger Collaboration, Astropart. Phys. 34, 368 (2011)

[7] I. Valiño for the Pierre Auger Collaboration, in Proc. of ICRC 2015 (Proceedings of Science, The Hague Netherlands, 2015)

[8] Pierre Auger and Telescope Array Collaborations, I.C. Maris et al., in Proc. of UHECR2014 (Utah USA, 2015)

[9] A. Yushkov for the Pierre Auger Collaboration, in these proceedings

[10] The Pierre Auger Collaboration, The Astrophysical Journal 804 (1), 15 (2015)

[11] IceCube, Telescope Array and Pierre Auger Collaborations, in Proc. of ICRC 2015 (Proceedings of Science, The Hague Netherlands, 2015) 
[12] The Pierre Auger Collaboration, The Astrophysical Journal 802(2), 111 (2015)

[13] A. Di Matteo for the Pierre Auger Collaboration, in Proc. of ICRC 2015 (Proceedings of Science, The Hague Netherlands, 2015) 0249

[14] R. Aloisio et al., JCAP 1210, 007 (2012)

[15] E. Armengaud et al., Astropart. Phys. 42, 463 (2007)

[16] S. Ostapchenko, Phys. Rev. D74, 014026 (2006)

[17] E.-J. Ahn et al., Phys. Rev. D80, 094003 (2009)

[18] K. Werner, F.-M. Liu, and T. Pierog, Phys. Rev. C74, 044902 (2006)

[19] D. Martello for the Pierre Auger Collaboration, Nucl. Instr. and Meth. A742, 16 (2014)
[20] R. Engel for the Pierre Auger Collaboration, in Proc. of ICRC 2015 (Proceedings of Science, The Hague Netherlands, 2015) 0686

[21] Pierre Auger Collaboration, The Pierre Auger Observatory Upgrade AugerPrime Preliminary Design Report, in arXiv: 1604.03637 [astro-ph.IM] (2015)

[22] Pierre Auger Collaboration, Journal of Instrumentation, 11, P02012 (2016)

[23] A. Letessier-Selvon et al., Nucl. Instrum. Meth. A767, 41 (2014)

[24] M. Ave et al., in Proc. of ICRC 2015 (Proceedings of Science, The Hague Netherlands, 2015) 0378 